\title{
Dragage en estuaire et milieu marin
}

\author{
Estuarine and marine dregging
}

par Claude Alzieu

IFREMER - Sète

Port activities require continuous or occasional dredging of considerable quantities of sediments : approximately 50 million $\mathrm{m}^{3}$ along the French coastline. A large proportion of the dredgings is authorized for dumping at sea in geographically delimited areas and following an impact evaluation of their potential harmfulness to the marine environment. The environmental risks assessment linked to dredging operations relies on knowledge related to several scientific disciplines.

\section{INTRODUCTION}

Les zones côtières, y compris les estuaires, sont le lieu d'activités multiples, parmi lesquelles la navigation tient une place importante. Pour permettre aux bateaux d'accéder aux structures portuaires il est alors indispensable de réaliser des dragages, au moment de la construction du port, mais également de façon périodique pour enlever les sédiments qui se sont accumulés dans les chenaux et les darses (dragages d'entretien). Le dragage constitue une activité vitale pour l'exploitation des ports. En France, environ 50 millions de $\mathrm{m}^{3}$ de sédiments sont dragués en moyenne par an et rejetés dans le milieu marin. Les trois principaux ports d'estuaires (Rouen, Nantes - St-Nazaire et Bordeaux) font état d'un volume moyen annuel d'environ 25 millions de $\mathrm{m}^{3}$, dont 6,5 millions de $\mathrm{m}^{3}$ de sables, 9,3 millions de $\mathrm{m}^{3}$ de vases clapées dans des zones d'immersion et une quantité équivalente rejetée en surverse. Les cinq grands ports maritimes, Dunkerque, Calais, Boulogne, le Havre et la Rochelle, draguent un volume moyen annuel de 6,2 millions de $\mathrm{m}^{3}$, composé par environ $20 \%$ de sables et $80 \%$ de vases. Les ports de la Méditerranée constituent des cas particuliers : Marseille n'effectue pratiquement pas de dragage d'entretien et en Languedoc-Rousillon les apports sableux maritimes constituent une entrave permanente aux accès portuaires. Toutefois, la contribution importante des grands ports d'estuaires aux volumes de matériaux rejetés en mer ne doit pas faire oublier les dragages, moins conséquents en volume, des multiples autres enclaves portuaires, qui peuvent poser des problèmes compte tenu des contraintes environnementales.

\section{LA PROBLÉMATIQUE ENVIRONNEMENTALE DES DRAGAGES}

En France le maintien de l'accès des navires aux zones portuaires est du domaine de responsabilité de l'Etat et exercée par les ports, sous la tutelle de la Direction du Transport maritime, des Ports et du Littoral, au Ministère de
l'Equipement, des Transports et du Logement. Du point de vue réglementaire, les lignes directrices pour l'immersion des déblais de dragages sont fixées au niveau international par la Convention d'Oslo. Même si la pratique des dragages n'est pas à remettre en cause, du fait des entraves à la navigation ou de l'incidence économique qui en résulteraient, elle ne peut être poursuivie qu'en prenant en considération les contraintes de préservation des écosystèmes côtiers. En effet, il est reconnu que les immersions sont une voie de transfert des contaminants vers le milieu marin; il convient donc de prendre les dispositions de nature à en limiter les impacts sur les écosystèmes. Les recommandations issues du «séminaire international sur les aspects environnementaux liés aux activités de dragages " (Nantes 27 novembre - $1^{\text {er }}$ décembre 1989 ) indiquent entre autres que :

- les impacts environnementaux ne pouvant être évités, il est important d'évaluer et de comparer les avantages des opérations de dragages aux conséquences prévisibles sur l'environnement :

- la nature et l'ampleur de la contamination des sédiments par des substances anthropiques déterminent largement la nécessité de mesures complémentaires destinées à prévenir le préjudice à l'environnement ou à la santé de l'homme ;

— à long terme, le problème des sédiments fortement pollués ne peut être résolu que par la suppression des sources de contamination ;

- le transport et la dispersion des matières en suspension constituent des facteurs essentiels pour la détermination de la nature et de l'ampleur des impacts environnementaux :

- pour l'élimination des sédiments faiblement contaminés le recours à des zones dispersives n'est pas dépourvu de risques environnementaux et exige l'étude du devenir et des effets des matériaux dispersés. Les zones de haute mer, situées en des points éloignés de la côte, constituent rarement sur le plan de l'environnement une solution souhaitable pour la prévention de la pollution marine ; 
- lorsqu'il existe un doute sérieux sur l'acceptabilité des options d'élimination en milieu aquatique, il conviendrait de procéder à une comparaison approfondie des impacts aquatiques avec ceux des solutions alternatives à terre (Alzieu et Gallenne, 1989).

Ainsi les problèmes auxquels ont à faire face les opérateurs des dragages, ainsi que les services chargés de la protection de l'environnement, relèvent à la fois de l'appréciation de l'impact potentiel des opérations et des solutions alternatives à l'immersion quand les sédiments présentent des caractéristiques toxiques indéniables. Cette problématique peut être traduite sous forme de trois questions principales pour lesquelles il n'existe pas de réponse unique et qui doivent être examinées au cas par cas :

- Comment prévoir les effets de la charge polluante lors des opérations de surverse ou d'immersion?

- Comment déterminer et suivre l'impact physique et écologique des rejets par clapage ou immersion?

-Que faire des sédiments réputés toxiques? Quelles sont les solutions écologiquement et économiquement acceptables ?

Les réponses à ces trois questions passent par des études d'évaluation des risques environnementaux, faisant appel à la modélisation mathématique du devenir des contaminants et de leur transport, ainsi qu'à une approche d'écotoxicologie expérimentale et de suivi des zones d'immersion.

\section{III $\square$ RISQUES ENVIRONNEMENTAUX}

\section{III.1 Impact global}

Les déblais de dragage non contaminés, tels que les sables, ne posent pas de problèmes particuliers quant à leurs effets sur l'environnement et sont même utilisés de façon courante pour la création de zones humides ou l'engraissement de plages en cours d'érosion. En revanche, le rejet de matériaux contaminés pose un certain nombre de questions relatives à l'impact sur la qualité physicochimique des eaux, au devenir des substances toxiques et à leurs effets sur les organismes vivants.

Cet impact est souvent malaisé à évaluer, faute de pouvoir disposer de données de suivi et d'une méthodologie adaptée et éprouvée. Ainsi, l'expérience tend à montrer que :

- les impacts des opérations de dragage et d'immersion des boues sur l'environnement côtier sont spécifiques du site où se déroulent ces opérations (Monbet, 1999),

- les effets de recouvrement des fonds par la charge solide sont faibles en dehors de la zone de dépôt,

- les apports de microorganismes d'origine anthropique (bactéries, virus) ne semblent pas affecter la salubrité des eaux au-delà de la zone de dépôt (Crenn et al., 1999),

- les opérations de dragage et d'immersion présentent un risque de dissémination géographique du dinoflagellé toxique Alexandrium minutum, par l'intermédiaire des formes enkystées présentes dans le sédiment. Ce risque est d'autant plus élevé que l'on se trouve à proximité de régions conchylicoles (Erard-le Denn, 1999).

\section{III.2 Evaluation des risques}

Les impacts des immersions sur la qualité physicochimique des eaux se traduisent essentiellement par des effets à court terme. Par contre, les contaminants adsorbés sur les particules peuvent subir des transformations diverses, dont certaines sont de nature à accroître leur biodisponibilité, donc leurs effets sur les organismes vivants.

\section{Niveaux de référence}

Différentes méthodes pour apprécier les risques potentiels sont utilisées. Elles consistent d'abord à faire référence à des critères de qualité des sédiments, déterminés par des seuils acceptables pour différents contaminants. Ainsi la Convention d'Oslo a défini deux niveaux qui s'appliquent à des contaminants désignés :

- niveau 1: valeurs en dessous desquelles l'immersion peut être autorisée sans études complémentaires et au dessus desquelles une étude plus approfondie pourrait être nécessaire :

— niveau 2 : valeurs au-delà desquelles l'immersion pourrait être interdite, sauf si l'immersion constitue la solution la moins dommageable pour l'environnement.

Sur la base de ces définitions l'arrêté interministériel du 14 juin 2000, publié au Journal Officiel du 10 août 2000, fixe les valeurs de référence pour les métaux et contaminants organiques (tableaux l et 2): il est complété par la circulaire interministérielle «relative aux conditions d'utilisation du référentiel de qualité des sédiments marins ou estuariens ». Cette dernière précise que ces seuils constituent des points de repère permettant de mieux apprécier l'incidence que peut avoir l'opération projetée. Ainsi, au-dessous du niveau NI, l'impact potentiel est en principe jugé d'emblée neutre ou négligeable, alors qu'entre le niveau $\mathrm{N} 1$ et le niveau $\mathrm{N} 2$, une investigation complémentaire peut s'avérer nécessaire. Audelà du niveau $\mathrm{N} 2$, on considère qu'il existe des indices notables laissant présager un impact potentiel négatif sur l'environnement. Une étude spécifique est alors requise, afin de déterminer la sensibilité du milieu aux substances concernées, la toxicité globale du sédiment et d'évaluer l'impact prévisible sur le milieu.

\section{Toxicité globale des sédiments}

Les niveaux de référence ne concernant qu'un nombre limité de contaminants il est parfois nécessaire de déterminer la toxicité globale du sédiment, à l'aide d'un ou plusieurs bioessais de laboratoire. Une étude comparative, conduite sur

Tableau 1 -Niveaux relatifs aux éléments traces (en $\mathrm{mg} / \mathrm{kg}$ de sédiment sec analysé sur la fraction inférieure à $2 \mathrm{~mm}$ ).

\begin{tabular}{|l|c|c|}
\hline Eléments traces & Niveau N1 & Niveau N2 \\
\hline Arsenic & 25 & 50 \\
\hline Cadmium & 1,2 & 2,4 \\
\hline Chrome & 90 & 180 \\
\hline Cuivre & 45 & 90 \\
\hline Mercure & 0,4 & 0,8 \\
\hline Nickel & 37 & 74 \\
\hline Plomb & 100 & 200 \\
\hline Zinc & 276 & 552 \\
\hline
\end{tabular}


Tableau 2 - Niveaux relatifs aux composés traces (en $\mathrm{mg} / \mathrm{kg}$ de sédiment sec analysé sur la fraction inférieure à $2 \mathrm{~mm}$ ).

\begin{tabular}{|l|c|c|}
\hline \multicolumn{1}{|c|}{ PCB } & Niveau N1 & Niveau N2 \\
\hline PCB Totaux & 0,5 & 1 \\
\hline PCB congénère 28 & 0,025 & 0,05 \\
\hline PCB congénère 52 & 0,025 & 0,05 \\
\hline PCB congénère 101 & 0,05 & 0,1 \\
\hline PCB congénère 118 & 0,025 & 0,05 \\
\hline PCB congénère 138 & 0,050 & 0,10 \\
\hline PCB congénère 153 & 0,050 & 0,10 \\
\hline PCB congénère 180 & 0,025 & 0,05 \\
\hline
\end{tabular}

des sédiments portuaires représentatifs des milieux portuaires français, a conduit à retenir les tests ci-après en raison de leur sensibilité et de leur aptitude à différencier les divers types de contamination :

— Embryotoxicité des œufs fécondés de bivalve: l'huître creuse (Crassostrea gigas) ou la moule (Mytilus edulis). La phase finale du développement embryonnaire des bivalves est constituée par le stade de larve « D ", qui correspond au début du stade larvaire. La toxicité des sédiments est alors évaluée par le pourcentage d'anomalies du développement embryonnaire pour des expositions à des concentrations en sédiment comprises entre 0 et $10 \mathrm{~g} / \mathrm{l}$ (Quiniou et al., 1997).

- Microtox® phase solide, méthode sensible basée sur la bioluminescence de la bactérie marine Phosphobacterium phosphoreum (ou Vibrio fischeri) qui peut être activée ou inhibée en présence de substances toxiques. Les résultats sont exprimés en pourcentages d'inhibition ou d'activation de la luminescence par rapport au témoin pour les faibles effets ou en CE50: Concentration Efficace entraînant une diminution de la bioluminescence de $50 \%$ par rapport à la valeur des témoins.

- Corophium sp., l'essai consiste à mettre en contact, pendant 10 jours, des amphipodes adultes de Corophium volutator ou $C$. arenarium avec les sédiments à tester, tels quels ou dilués avec celui d'où proviennent les animaux. Au cours des 10 jours de contact, les amphipodes morts sont dénombrés et retirés, en fin d'expérience. Les résultats sont exprimés en CL20 calculée sur la base du sédiment $\sec (\mathrm{g} / \mathrm{l})$.

- Tigriopus brevicornis, cette méthode, qui dérive du protocole normalisé ISO / DIS.14 669 pour les copépodes marins, est destinée à déterminer la toxicité des substances chimiques solubles, des effluents et des eaux de mer ou d'estuaire. Le taux de mortalité de $50 \%$ des copépodites est alors exprimé en grammes de sédiment sec par litre. Ce test peut être adapté pour mettre en évidence des effets sublétaux (Forget et al., 1998).

\section{GEODRISK}

Pour faciliter l'évaluation des risques des sédiments contaminés, le Groupe d'Etude et d'Observation sur les Dragages et l'Environnement (GEODE) a réalisé un logiciel d'aide à la décision, GEODRISK, basé sur les résultats d'analyses chimiques recommandées par les "instructions techniques portant sur le prélèvement et l'analyse des déblais de dragage " en date du 14 juin 2000 .

La démarche suivie consiste, suivant la méthode des scores de risques, à prendre successivement en considération les domaines suivants :

- le danger potentiel de chaque contaminant analysé, à partir de leur affinité pour la phase dissoute, de leur toxicité intrinsèque, de leur capacité de bioaccumulation dans les chaînes alimentaires :

— la toxicité globale du sédiment obtenue à partir des tests de laboratoire :

- la potentialité du transfert des contaminants à partir de la zone de dépôt :

— la sensibilité de l'écosystème récepteur.

- Pour chacun des domaines ci-dessus sont définis des critères spécifiques, auxquels sont attribués des scores de risques, fixés à partir de données bibliographiques sur la biogéochimie et l'écotoxicologie des contaminants considérés. GEODRISK permet ainsi de discriminer les sédiments dragués, selon leurs niveaux de contamination, leurs toxicités potentielles et mesurées (Alzieu et Quiniou, 2001). Un arbre de décision permet aux gestionnaires d'orienter l'analyse du dossier vers trois propositions possibles:

1) Rejet ou immersion en zone autorisée.

2) Réalisation d'une étude locale d'impact, qui peut être considérée comme le résultat de toutes les connaissances acquises sur le site d'immersion. C'est à ce stade que sera prise en considération la sensibilité de la zone d'immersion envisagée en regard, d'une part des risques écotoxicologiques et, d'autre part des risques sanitaires induits par la contamination bactériologique des sédiments ou la présence de kystes de dinoflagellés toxiques (Alexandrium minitum). Dans certains cas cette évaluation peut être particulièrement complexe et faire appel, par exemple, à des modèles numériques de dispersion locaux ou régionaux pour évaluer le transport des matériaux hors de la zone d'immersion.

3) Réalisation d'une étude d'impact approfondie qui doit, par référence aux prescriptions réglementaires, apporter la preuve que l'immersion constitue la solution la moins préjudiciable pour l'environnement. Le terme environnement doit être pris dans son sens le plus large et inclure, par exemple, une évaluation de l'impact terrestre dans le cas ou le dépôt ou traitement à terre constituerait une solution alternative envisagée. Les lignes directrices de la Convention OSPAR pour la protection du milieu marin de l'Atlantique du Nord-Ouest et du Protocole relatif à la Protection de la Méditerranée, listent les critères à prendre en considération pour l'évaluation des effets potentiels et de la nature des impacts.

\section{IV $\square$ CONCLUSION}

Les millions de $\mathrm{m}^{3}$ de sédiments dragués annuellement dans les ports proviennent, soit de l'entretien régulier des chenaux, soit d'opérations de restauration des niveaux d'eau dans des darses ou des bassins plus ou moins exposés aux apports polluants. Il en résulte une contamination variable des sédiments qui nécessitent des procédures de gestion adaptées. Les conventions internationales ont défini des lignes directrices applicables à la gestion des matériaux de dragage et 
proposé de meilleures pratiques environnementales (BEP) qui visent à minimiser les effets causés par l'immersion, réduire les quantités immergées et améliorer la qualité des sédiments par des mesures préventives. L'immersion étant une pratique généralisée et économique, les conventions ont recommandé des procédures type à suivre pour la délivrance des autorisations. La démarche d'ensemble consiste à sélectionner les sédiments qui, par leur nature et leur charge polluante, pourraient présenter des risques écologiques pour les écosystèmes côtiers.

\section{BIBLIOGRAPHIE}

[1] Alzieu Cl., Galıenne B. (1989), - Actes du séminaire international sur les aspects environnementaux liés aux activités de dragages - Nantes 27 novembre-1 ${ }^{\text {er }}$ décembre 1989.

[2] Alzieu Cl., Quiniou F. (2001). - GEODRISK : La démarche d'analyse des risques liés à l'immersion des boues de dragage des ports maritimes. CDROM Editions IFREMER, Plouzané.

[3] Arrêté relatif aux niveaux de référence à prendre en compte lors d'une analyse de sédiments marins ou estuariens présents en milieu naturel ou portuaire : Publié au Journal Officiel le 10 août 2000.
[4] Circulaire relative aux conditions d'utilisation du référentiel de qualité des sédiments marins ou estuariens présents en milieu naturel ou portuaire défini par arrêté interministériel : Publié au Journal Officiel le 10 août 2000.

[5] Crenn I., Gourmelon M., Le Cann P., Ménard D., Le Guyader F. Derrien A. Pommeruy M. (1999). - Microbiologie sanitaire des sédiments. In Dragage et environnement marin: état des connaissances, Alzieu Cl. Coordinateur, Editions IFREMER, Plouzané. 223 p.

[6] Erard-Le Denn E. (1999). - Phytoplancton toxique et sédiments. In Dragage et environnement marin : état des connaissances, Alzieu Cl. Coordinateur, Editions IFREMER, Plouzané. $223 \mathrm{p}$.

[7] Forget J., Menasria M.R., Pavillon J.F., Bocquene G. (1998). Mortality and LC values for several stages of the marine copepod Tigriopus brevicornis (Müller) exposed to metals arsenic and cadmium and the pesticides atrazine, carbofuran, dichlorvos and malathion. Ecotox. Environ. Saf, 40, 239-244.

[8] Monbet Y. (1999), - Les dragages et leurs impacts sur l'environnement. In Dragage et environnement marin: état des connaissances, Alzieu Cl. Coordinateur, Editions IFREMER, Plouzané, 223 p.

[9] Quiniou F., Judas A., Le Squer-André E. (1997). - Toxicité potentielle des eaux des sédiments des principaux estuaires de la rade de Brest évaluée par deux bioessais. Ann. Inst. Océanogr. Paris, 73, 1, 35-48. 